\title{
Pulmonary sclerosing pneumocytoma, an adenocarcinoma mimicker: A case report and review of the literature
}

Mohd Zulkimi Roslly, M.D.

Aida Widure Mustapha Mohd Mustapha, M.D.

Nik Farhan Nik Fuad, M.D.

From Department of Radiology, Faculty of Medicine, Universiti Kebangsaan Malaysia , Malaysia.

Address correspondence to M.Z.R. (e-mail: zulkimiroslly@gmail.com)

Received 18 February 2021; revised 17 April 2021; accepted 17 April 2021

doi:10.46475/aseanjr.v22i1.120

\section{Abstract}

Pulmonary sclerosing pneumocytoma (PSP) is a rare condition, occupying only approximately $1 \%$ of all benign lung neoplasms and the description of this disease through a radiological perspective is limited. Only approximately a third of PSP has been correctly diagnosed pre-operatively through contrast-enhanced computed tomography (CECT) thorax. To date, only few radiological features have been described to ascertain this disease and there is still a significant portion of patients being misdiagnosed with a malignant neoplasm. We report a case of PSP that was initially misdiagnosed as lung adenocarcinoma stage IIA and the histopathological findings of surgical resection confirmed the diagnosis of PSP. We try to add some points from this case into the previous radiological description of this uncommon disease with a common presentation.

Keywords: Sclerosing, Pneumocytoma, Adenocarcinoma, Pulmonary. 


\section{Introduction}

Pulmonary sclerosing pneumocytoma (PSP), previously known as pulmonary sclerosing hemangiomas (PSH) is rare, accounting for approximately $1 \%$ of all benign lung tumours. It was first described in 1956 by Liebow and Hubbell [1] after several personal observations and analyses of similar cases being reported under different entities including xantoma, histiocytoma and xanthofibroma. Subsequently, the term "sclerosing hemangioma" was selected based on the identical histologic morphology until year 2015 where the World Health Organization (WHO) changed the term "sclerosing hemangioma" to "sclerosing pneumocytoma" under the new classification of lung tumours, after several immunohistochemistry studies [2]. This entity predominantly affects middle-age adults of over 50 years old with 5:1 female to male ratio predilection [3]. To date, only a few radiological features have been described to ascertain this disease and there is still a significant portion of patients being misdiagnosed with a malignant neoplasm. We report a case of PSP that was initially misdiagnosed as lung adenocarcinoma stage IIA and the histopathological findings of surgical resection confirmed the diagnosis of PSP.

\section{Case summary}

A 60-year-old lady with hypertension and type 2 diabetes was physically well except mild occasional shortness of breath on exertion. Physical examinations were unremarkable. A routine chest radiograph (CXR) was performed during a regular follow-up for her premorbid condition in a health clinic showed a right lung mass. She was then subject to contrast-enhanced computed tomography (CECT) of the thorax for further evaluation.

The initial chest radiograph (Figure 1 ) and CECT of the thorax (Figure $2 \mathrm{a}$ and $2 \mathrm{~b}$ ) revealed a large well-defined heterogeneous rounded mass at the right perihilar region in the anterior segment of the right upper lobe measuring $6.0 \times 6.5 \times 5.8 \mathrm{~cm}$. The mass displaced the right minor fissure inferiorly and splayed the adjacent vessels and bronchi of the right hilum. There was no evidence of distant metastasis. 


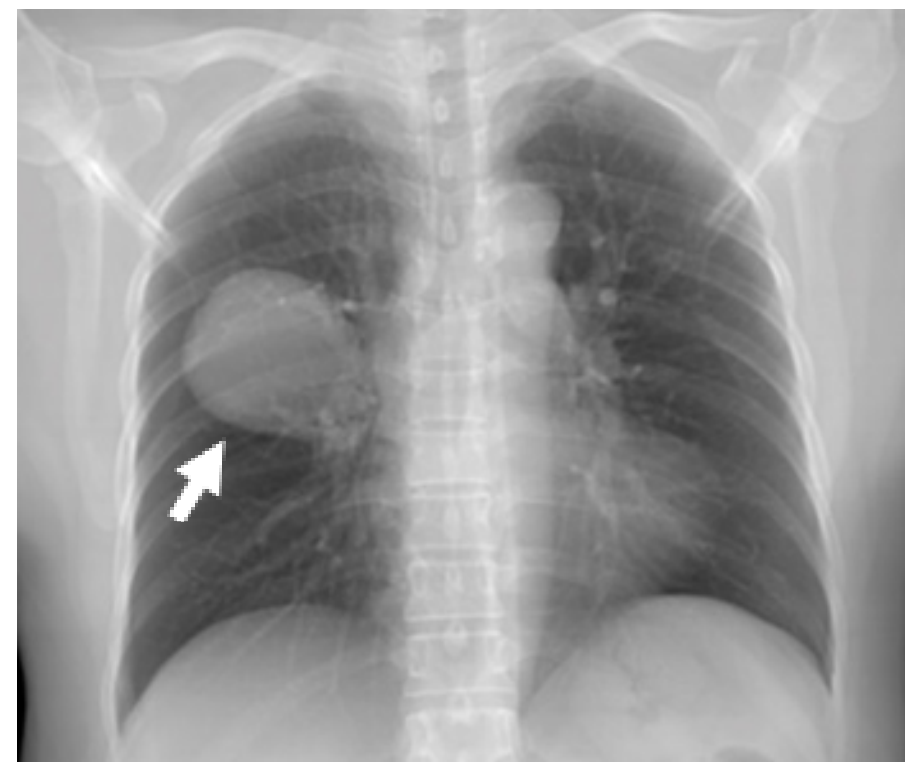

Figure 1. Frontal chest radiograph reveals a well-defined mass at right perihilar region (arrow).

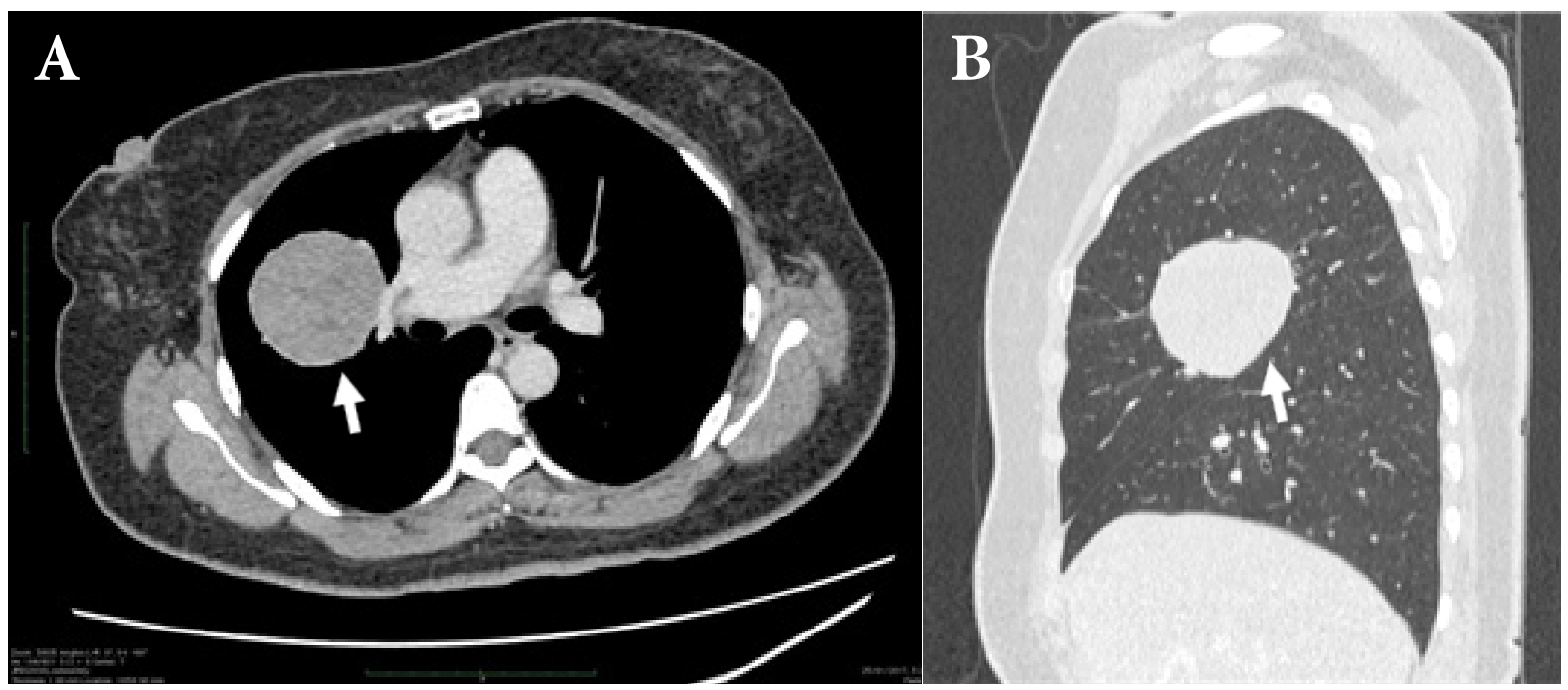

Figure 2. Axial image of contrast-enhanced CT (a) and sagittal image in lung window (b) show a well-defined heterogenously enhancing lung mass at right perihilar region. (arrows). 
Bronchoscopy showed the anterior segment of the right upper lobe bronchus slightly compressed by mass but did not obstruct the lumen and there was no endobronchial mass detected. A core-needle biopsy was performed under the CT-guidance and the histopathological report showed lung adenocarcinoma stage IIA. The core biopsy showed positivity towards thyroid transcription factor-1 (TTF-1) but proved inconclusive for cytokeratin-7 (CK7). Cytokeratin-20 (CK 20) stain was not available in the laboratory; however, the overall conclusion of the core biopsy being adenocarcinoma in the right lung was made based on the TTF-1 positivity. She developed chesty cough and constitutional symptoms a few weeks later and was treated accordingly.

CECT of the thorax, abdomen and pelvis was performed four months later for restaging which showed a well-defined heterogenous perihilar lung parenchymal mass in the anterior segment of the right upper lobe with no significant change in size as the previous CECT findings. The lesion was located $5.2 \mathrm{~cm}$ from the carina and caused splaying with mild narrowing of the right upper bronchus and bronchus intermedius with the patent bronchial lumen. Postero-inferiorly, it was abutting the right oblique fissure. It showed average attenuation of $+44 \mathrm{HU}$ with a maximum enhancement of $+57 \mathrm{HU}$. There was no fat-attenuating component or calcification shown within the mass. The presence of subcentimeter right paratracheal lymph node was detected. The overall CECT findings concluded that this patient was in stage T2N0Mx of lung carcinoma. Fluorine-18 fluorodeoxyglucose positron emission tomography CT $\left({ }^{18} \mathrm{~F}-\mathrm{FDG}\right.$ PET/CT) was also performed for complete assessment. It showed a heterogenously hypermetabolic mass with $\mathrm{SUV}_{\max }$ of 5.5. Core-needle biopsy was repeated. The overall histopathological study reported adenocarcinoma of the right lung. 


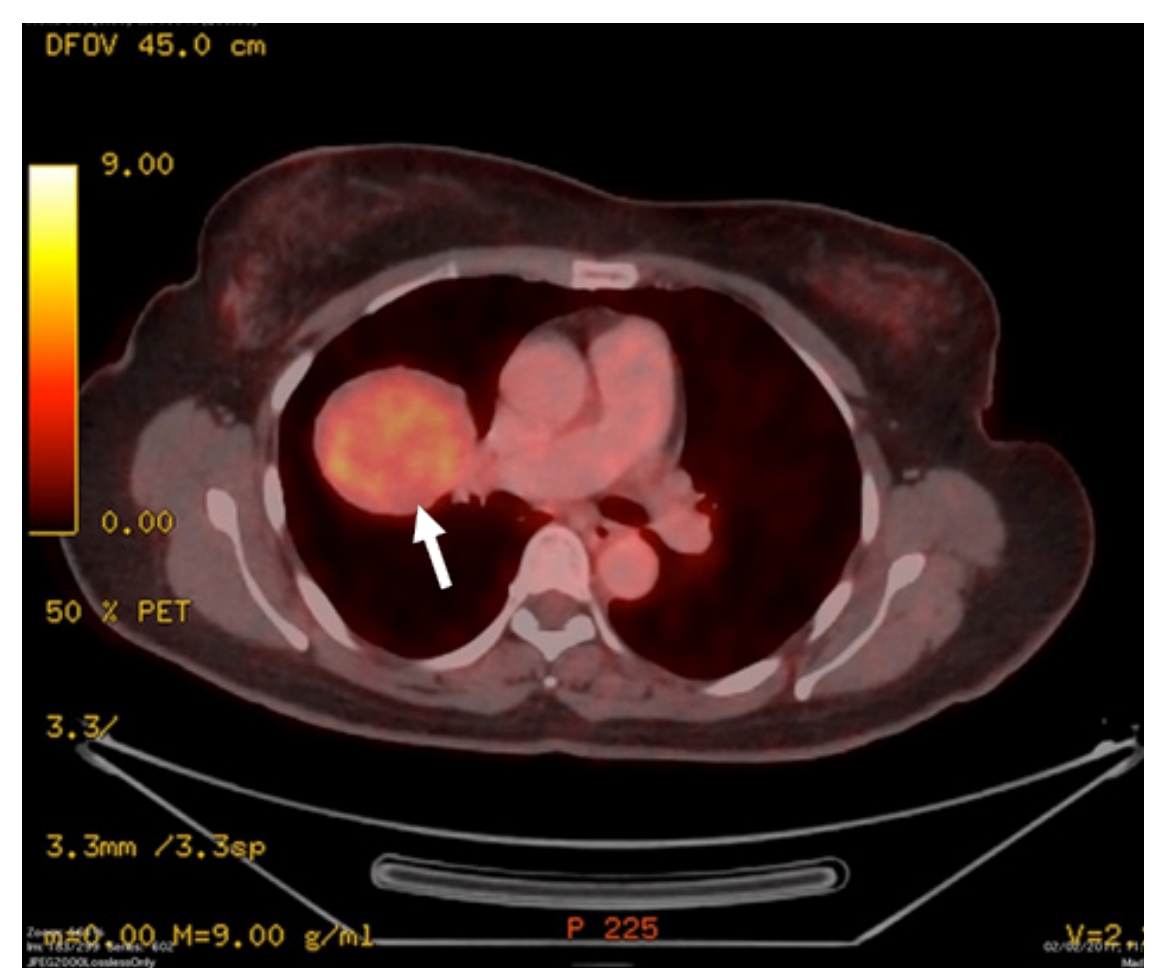

Figure $3 .{ }^{18} \mathrm{~F}-\mathrm{FDG}$ PET/CT image in axial view shows a heterogenously hypermetabolic right perihilar mass with SUV $\max$ of 5.5 (arrow).

The patient then was subject to thoracotomy and bilobectomy of the right upper and middle lobes. Intraoperatively, approximately $6 \mathrm{~cm}$ of haemorrhagic tumour was noted at the right upper lobe invading the middle lobe. The adjacent lymph nodes were also sampled. Surgical specimens were seen $15 \mathrm{~mm}$ from the nearest bronchial margin and $10 \mathrm{~mm}$ from the parenchymal margin. Microscopically, it showed a circumscribed tumour comprising two populations of neoplastic cells: the cuboidal surface cells and the stromal round cells. Also, mitotic figure was rarely seen (Figure 4). Further immunohistochemistry showed the final diagnosis of sclerosing pneumocytoma in the right lung. 


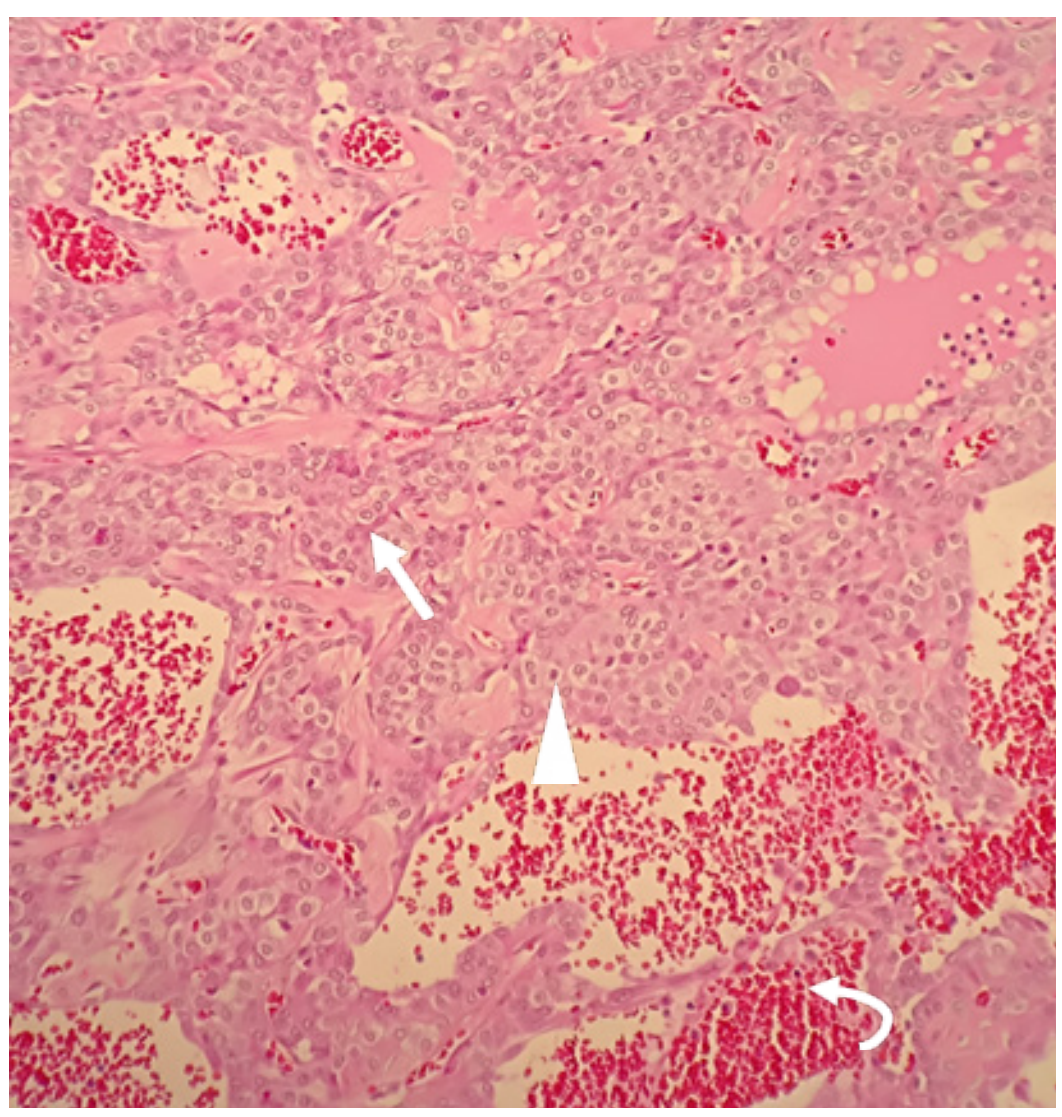

Figure 4. Hematoxylin and Eosin staining of the surgical specimen shows two populations of neoplastic cells: the cuboidal surface cells (arrow) and the stromal round cells (arrowhead) with patchy areas of the hemorrhagic component (curved arrow).

The patient was diagnosed with hospital-acquired pneumonia post-operatively but discharged well 17 days post-op after being treatment. Currently, the patient is doing well at home and asymptomatic. 


\section{Discussion}

PSP was mainly found incidentally in around $70 \%$ of the patients $[2,4,5]$. Referring to our case, the mass was found during a regular follow up chest radiograph. A smaller number of patients may present with non-specific symptoms like hemoptysis, chronic cough, and chest pain.

The most common presenting form is an asymptomatic solitary pulmonary nodule that can grow up to $7 \mathrm{~cm}$ in diameter. Although $73 \%$ of the lesions are smaller than $3 \mathrm{~cm}$ [2], PSP is usually found as a moderate-size lesion with the diameter sizes of the tumours ranging from 2.5-6.5 cm [6]. Most patients manifest with a single lesion, accounting for $92.1 \%$ as reported by Shin SY et al. [4]. This finding is consistent with a study done by Wang et al. [7] whereby $100 \%$ of the patients had a single pulmonary lesion. Yang Lei et al. [8] reported 96\% of the patients found to have a solitary lung mass irrespective of the location which were mainly round in shape. Our case appeared to be compatible with the findings in both of these studies.

In a study by Cheung YC et al.[6], all of the PSP appeared to be located in the perihilar region with all lesions smoothly encapsulated. He also reported that all PSP appeared to be located at the perihilar region. In a study by Wang QB et al [7], PSP appeared as a well-defined mass and was generally a juxtapleural mass in $94 \%$ of the patients on the CT. As found by Wang Q.B [7] and Im JG et al. [9], the PSP was found as a juxtapleural mass in $94 \%$ and $100 \%$ of the patients, respectively. Our case showed that the mass was located $5.2 \mathrm{~cm}$ from the carina based on the CECT thorax and lay $1.0 \mathrm{~cm}$ from the parenchymal margin.

According to Cheung Y et al. [6], in terms of the contrast enhancement pattern, the majority of the patients had heterogeneous enhancement with $\mathrm{HU}$ ranging from +67 to $+112 \mathrm{HU}$. The mean CT attenuation of the lesions from 76 patients was $+76(+/-47.1)$ (range, 0-150.3) HU. Heterogeneous attenuation was more common (59.0\%) in CECT studies [4]. In our case, the enhancement pattern of the mass was fairly heterogenous. The average attenuation of the mass was 
$+44 \mathrm{HU}$; with the minimum and maximum enhancement attenuation of $+40 \mathrm{HU}$ and $+57 \mathrm{HU}$, respectively which corresponded to the studies conducted earlier. However, the non contrast-enhanced study was not performed in our case according to the local scanning protocol. There was no area with fat-attenuation or calcification within the mass. Neither were there areas of fluid-attenuation to suggest a liquified or necrotic component. In contrast to a study by Wang QB et al.[7], the attenuation of post enhancement was found to be higher at $+79(+/-3)$ HU and homogenous in $75 \%$ of the patients. They also concluded that the diameter of the heterogeneously enhanced tumour was larger than that of the homogenously enhanced tumours. On a CT-pathology correlation, the higher, equal, or lower attenuation areas correspond with angiomatous, solid and sclerotic, and cystic areas, respectively [9].

Based on a relatively large retrospective study by Shin SY et al. [4] of 76 patients with confirmed of PSP, 4 specific CT signs were identified. They are marginal pseudocapsule sign, overlying vessel sign, air-gap sign and halo sign. However, none of these signs were present in our case. The majority of patients presented a peripheral, solitary lesion with smooth margins [8].

PSP classically is a histological diagnosis and correct diagnosis on the formal radiologic report of the conventional chest CECT is possible in only $30.3 \%$ of 76 patients [5]. Its CECT manifestation varies according to the composition [6].

${ }^{18}$ F-FDG PET hypometabolic uptake of PSP mass was evident in a study by Shin SY et al. [4] that showed 17 patients who underwent ${ }^{18} \mathrm{~F}$-FDG PET showing a hypo- or intermediate metabolic lesion. This was in contrast to several studies performed on the FDG uptake of PSP such as by Lin KH et al. [10] where they found that the maximal standard uptake value (SUVmax) of FDG uptake was positively correlated with the tumour size. This shows that a larger PSH has a higher uptake and might be misrecognized as a malignancy. In our case, the mass was considered a large mass $(>5 \mathrm{~cm})$, thus, demonstrating a hypermetabolic activity on ${ }^{18} \mathrm{~F}-\mathrm{FDG}$ PET. 


\section{Conclusion}

We report a case of PSP which was initially diagnosed as lung adenocarcinoma. PSP is a rare benign lung tumour and tissue diagnosis is the gold standard investigation. The radiological description and diagnosis remain difficult and there is no definitive diagnostic radiological finding of PSP which might lead to misdiagnosis and unnecessary surgical intervention with the patient. However, the clinical and radiological characteristics of the mass described in our case and in the literature may guide the reporting radiologist to infer this diagnosis in the list of differential diagnosis. 


\section{References}

1. Liebow AA, Hubbell DS. Sclerosing hemangioma (histiocytoma, xanthoma) of thelung. Cancer 1956;9:53-75. doi:10.1002/1097-0142(195601/02)9:1<53::aidcncr2820090104>3.0.co;2-u.

2. Devouassoux-Shisheboran M, Hayashi T, Linnoila RI, Koss MN, Travis WD. A clinicopathologic study of 100 cases of pulmonary sclerosing hemangioma with immunohistochemical studies: TTF-1 is expressed in both round and surface cells, suggesting an origin from primitive respiratory epithelium. Am J Surg Pathol 2000 ;24:906-16. doi: 10.1097/00000478-200007000-00002.

3. Neuman J, Rosioreanu A, Schuss A, Turi G, Yung E, Trow TK, et al. Radiologypathology conference: sclerosing hemangioma of the lung. Clin Imaging 2006;30:409-12. doi: 10.1016/j.clinimag.2006.05.030.

4. Shin SY, Kim MY, Oh SY, Lee HJ, Hong SA, Jang SJ, et al. Pulmonary sclerosing pneumocytoma of the lung: CT characteristics in a large series of a tertiary referral center. Medicine (Baltimore) 2015;94:e498. doi: 10.1097MD.0000000 000000498 .

5. Sugio K, Yokoyama H, Kaneko S, Ishida T, Sugimachi K. Sclerosing hemangioma of the lung: radiographic and pathological study. Ann Thorac Surg 1992 ;53:295-300. doi: 10.1016/0003-4975(92)91336-8.

6. Cheung YC, Ng SH, Chang JW, Tan CF, Huang SF, Yu CT. Histopathological and CT features of pulmonary sclerosing haemangiomas. Clin Radiol 2003 ;58:630-5. doi: 10.1016/s0009-9260(03)00177-6.

7. Wang QB, Chen YQ, Shen JJ, Zhang C, Song B, Zhu XJ, et al. Sixteen cases of pulmonary sclerosing haemangioma: CT findings are not definitive for preoperative diagnosis. Clin Radiol 2011;66:708-14. doi: 10.1016/j.crad. 2011.03.002. 
8. Lei Y, Yong D, Jun-Zhong R, Zhi Y, Zi-Tong W. Treatment of 28 patients with sclerosing hemangioma (SH) of the lung. J Cardiothorac Surg 2012;7:34. doi: 10.1186/1749-8090-7-34.

9. Im JG, Kim WH, Han MC, Han YM, Chung JW, Ahn JM, et al. Sclerosing hemangiomas of the lung and interlobar fissures: CT findings. J Comput Assist Tomogr 1994;18:34-8. doi: 10.1097/00004728-199401000-00007.

10. Lin KH, Chang CP, Liu RS, Wang SJ. F-18 FDG PET/CT in evaluation of pulmonary sclerosing hemangioma. Clin Nucl Med 2011;36:341-3. doi: 10.1097/RLU.0b013e31820aa00c. 